\title{
Association between the endogenous retrovirus HRES-1 and multiple sclerosis in the United Kingdom - evidence of genetically different disease subsets?
}

\author{
H.B. Rasmussen ${ }^{\text {a }}$, M.A. Kelly ${ }^{\mathrm{b}}$, \\ D.A. Francis ${ }^{\mathrm{c}}$ and J. Clausen ${ }^{\mathrm{a}}$ \\ ${ }^{a}$ Department of Life Sciences and Chemistry, Roskilde \\ University, PO Box 260, DK-4000 Roskilde, Denmark \\ ${ }^{\mathrm{b}}$ Department of Medicine, Division of Medical \\ Sciences, University of Birmingham, Edgbaston, \\ Birmingham B15 2TT, United Kingdom \\ ${ }^{\mathrm{c}}$ Queen Elizabeth Neurosciences Centre, Edgbaston, \\ Birmingham B15 2TH, United Kingdom
}

Received 1 May 2000

Accepted 28 June 2000

In the present study we determined the frequencies of four haplotypes of the human T-cell lymphotropic virus-related endogenous sequence, HRES-1, in 110 multiple sclerosis (MS) patients and 100 healthy control subjects from the United Kingdom. We found evidence of an association between this endogenous retrovirus and MS $(p<0.01)$, in particular reflecting an increased frequency of HRES-1 haplotype 1 in the group of patients. There was no significant difference in the distribution of HRES-1 haplotypes between relapsingremitting MS and the primary progressive form of the disease. The odds ratio for HRES-1 haplotype 1 and MS did not differ significantly between individuals positive for HLA-DR2 and DR2-negative individuals. Comparison of the observations from the present study with previous results implicated HRES-1 as a marker of genetic heterogeneity in MS.

Keywords: Multiple sclerosis, endogenous retrovirus, genetic susceptibility factor

\section{Introduction}

A considerable number of genes have been associated with multiple sclerosis (MS), a demyelinating disease of presumed autoimmune etiology, but in most cases these genetic associations have not been repro- ducible [3]. One of the reasons for this could be that several genes perhaps interacting with each other are involved, implying that the contribution from the single susceptibility locus is small. Moreover, there is evidence that the disease consists of several genetically distinct subgroups as suggested by the occurrence of different clinical phenotypes.

In Caucasians from Northern Europe and North America, HLA-DR2 has been associated with increased susceptibility to MS [3]. On examination of MS patients from other populations, absence of association with DR2 $[4,5]$ or associations with other HLADR markers [8] have been found, implying that distinct genes predispose to the disease in populations of different ethnic origin. Recent observations from the United Kingdom (UK) have provided evidence that MS patients positive for DR2 do not share susceptibility loci with DR2-negative MS patients, suggesting existence of distinct disease subtypes with DR2 being a marker of one of these [2]. The identification of two different types of MS in Japan, namely a DR2 associated Western type and an optico-spinal form or Asian type with no HLA-DR association, provides further evidence of genetic heterogeneity in this disease [6].

Endogenous retroviruses are normal genomic constituents some of which appear to be involved in the immune regulation by producing an immunosuppressive factor [15]. Others perhaps encode for proteins which act as autoantigens under certain circumstances [17]. This includes the human endogenous retrovirus HRES1 [1]. Consequently, endogenous retroviruses are potential candidates in the search for genetic susceptibility factors in MS and other diseases of presumed autoimmune etiology [11,17].

Previously, we have reported an association between MS and a haplotype of HRES-1 defined on the basis of single nucleotide polymorphisms in the promoter region and a synonymous nucleotide substitution in the 
segment of this endogenous retrovirus encoding a 28 $\mathrm{kDa}$ protein $[10,13]$. In addition a promoter allele of HRES-1 has been associated with protection against systemic lupus erythematosus [7]. Whether the genetic variation in the promoter affects the expression of the $28-\mathrm{kDa}$ protein, which can act as an autoantigen and perhaps contribute to the development of autoimmune diseases is not known. In the present study we searched for an association between MS and HRES-1 in individuals from the UK conditioning for the presence of HLA-DR2.

\section{Materials and methods}

\subsection{Patients and control subjects}

A total of $110 \mathrm{MS}$ patients and 100 healthy control subjects were recruited from the West Midlands region of the UK. All patients and control subjects were unrelated. The diagnosis of MS was made according to the Poser criteria [9]. Eighty-eight patients had relapsingremitting MS, while 22 patients were afflicted with primary progressive MS. The former group included 16 patients with secondary progressive MS, characterized by progression of symptoms after initial relapses and remissions. Patients as well as control subjects were white Caucasians. They had all been typed for HLADR and -DQ alleles in a previous study with results typical of a Northern European Caucasian population [5], i.e. $61 \mathrm{MS}$ patients and 30 healthy control subjects were DR2-positive corresponding to phenotypic frequencies of 0.55 and 0.30 , respectively. The haplotype DRB1*1501, DQA1*0102, DQB1*0602 was present in all DR2-positive individuals except two, namely a patient carrying the DRB $1 * 1502$ allele and a control subject with DRB1*1601.

\subsection{Genotyping of HRES-1}

Genotyping at three polymorphic sites of HRES-1 were carried out by enzymatic amplification and treatment of amplified products with HindIII, Eco57I and $N c i$, after which haplotypes were deduced on the basis of the known allelic associations [13].

\subsection{Analysis of data}

Genotypic proportions in the group of control subjects were tested for Hardy-Weinberg equilibrium using the program made by John Brzusto, University of Al- berta, Canada (http://www.biology.ualberta.ca/jbrzusto /hwenj.html). For linkage disequilibrium calculations the EH program was taken into use [16]. The CLUMP program was used to assess the significance of markerdisease associations [14]. Based upon "raw" 2-by-m tables with $m$ being the number of haplotypes, this program calculates a normal chi-squared value $\left(\mathrm{T}_{1}\right)$. The CLUMP program also tests each individual haplotype against a pool of all the others and lists that particular haplotype with the largest chi-squared value $\left(\mathrm{T}_{3}\right)$. Moreover, it permits the detection of an association between a disease and a variable number of haplotypes $\left(\mathrm{T}_{4}\right)$. For comparative purposes previous observations of HRES-1 haplotype distributions in MS patients and control subjects from Denmark were included [13]. Assessment of homogeneity of odds ratios in a stratified $2 \times 2$ table was done using the Breslow-Day test, implemented in the SAS System Version 6.12 (SAS Institute Inc., Cary, NC, USA).

\section{Results}

The genotypic frequencies at the HindIII, Eco57I and NciI polymorphic sites in the group of control subjects did not deviate significantly from with those expected under conditions of Hardy-Weinberg equilibrium as determined by the appearance of $p$ values of $0.67,0.59$, and 0.68 , respectively. The alleles at the three polymorphic sites were arranged into four different haplotypes (Table 1). On testing a model with this pattern of allelic association against the null hypothesis of no linkage disequilibrium, we found a $p$ value $<10^{-7}$ in patients as well as control subjects.

The distribution of the four HRES-1 haplotypes differed significantly between MS patients and control subjects from the UK, in particular due to an increased frequency of haplotype 1 among the patients. There was no significant difference in the HRES-1 haplotype distribution between healthy control subjects from the UK and Denmark whereas a significant difference was found on comparison of patients from these two countries. This difference was due to increased frequencies of haplotype 1 and 4 combined with decreased frequencies of haplotype 2 and 3 in the group of British patients.

The HRES-1 haplotypes of the British patients and control subjects were categorized into two groups, namely haplotype 1 and all others (haplotype 2, 3 and 4). Odds ratio for MS and HRES-1 haplotype 1 was 1.24 in DR2-positive individuals and 2.00 in DR2- 
Table 1

Distribution of HRES-1 haplotypes in multiple sclerosis patients and healthy individuals

\begin{tabular}{|c|c|c|c|c|c|c|c|}
\hline \multirow[t]{2}{*}{ Haplotypes $^{\mathrm{c}}$} & \multirow[t]{2}{*}{ HindIII } & \multirow[t]{2}{*}{ Eco57I } & \multirow[t]{2}{*}{$N c i \mathrm{I}$} & \multicolumn{2}{|c|}{ United Kingdom $^{\mathrm{a}}$} & \multicolumn{2}{|c|}{ Denmark $^{b}$} \\
\hline & & & & MS patients ${ }^{\mathrm{d}}$ & Control subjects ${ }^{\mathrm{d}}$ & MS patients $^{\mathrm{d}}$ & Control subjects ${ }^{\mathrm{d}}$ \\
\hline 1 & - & + & - & $94(0.43)$ & $60(0.30)$ & $47(0.30)$ & $85(0.38)$ \\
\hline 2 & + & + & + & $53(0.24)$ & $72(0.36)$ & $59(0.38)$ & $63(0.28)$ \\
\hline 3 & - & - & - & $13(0.06)$ & $18(0.09)$ & $17(0.11)$ & $13(0.06)$ \\
\hline 4 & - & + & + & $60(0.27)$ & $50(0.25)$ & $33(0.21)$ & $61(0.28)$ \\
\hline
\end{tabular}

${ }^{\text {a }}$ Data from this study.

${ }^{\mathrm{b}}$ Data from a previous study [13].

${ }^{\mathrm{c}}$ The haplotypes are defined on the basis of presence or absence of three restriction enzyme polymorphisms as indicated by + and - , respectively [13].

${ }^{\mathrm{d}}$ Numbers of haplotypes with frequencies in brackets.

MS patients from the UK $v s$ control subjects from the UK: chi-squared value of the "raw" table $\left(\mathrm{T}_{1}\right)=11.18, p=0.009$, chi-squared value of haplotype 1 against the rest $\left(\mathrm{T}_{3}\right)=7.34, p=0.03$, chi-squared value of haplotype 1 and 4 together against the rest $\left(\mathrm{T}_{4}\right)=10.10, p=0.006$.

Control subjects from the UK $v s$ control subjects from Denmark: chi-squared value of the "raw" table $\left(\mathrm{T}_{1}\right)=5.68$, $p=0.13$.

MS patients from the UK vs MS patients from Denmark: chi-squared value of the "raw" table $\left(\mathrm{T}_{1}\right)=13.87, p=0.002$; chi-squared value of haplotype 2 against the rest $\left(\mathrm{T}_{3}\right)=8.23, p=0.02$, chi-squared value of haplotype 1 and 4 together against the rest $\left(\mathrm{T}_{4}\right)=13.61, p=0.001$.

negative individuals. The Breslow-Day test did not provide evidence against the hypothesis of equal odds ratios in these two strata (Table 2).

The frequencies of haplotype 1-4 in patients with relapsing-remitting MS from the UK were $0.43,0.21$, 0.07 and 0.29 , respectively. These haplotypes were present at frequencies of $0.43,0.37,0.02$ and 0.18 in patients with primary progressive MS. Comparison of the haplotype distributions of these two MS subgroups revealed a chi-squared value $\left(\mathrm{T}_{1}\right)$ of 5.89 , corresponding to a $p$ value of 0.12 .

\section{Discussion}

In the present study we have searched for an association between HRES- 1 and MS in the UK. We found that the frequency of one of the HRES-1 haplotypes, namely haplotype 1 , was significantly higher in patients than healthy control subjects. The strength of this association did not differ significantly between DR2-positive and DR2-negative subjects. The lack of a significant difference in the HRES-1 haplotype distribution between relapsing-remitting MS and primary chronic progressive MS was in agreement with observations from Denmark [13].

The detection of an increased frequency of haplotype 1 in MS patients from the UK as compared with control subjects from the same country was unexpected since this haplotype was less frequent in MS patients than control subjects from Denmark. Further, there was a tendency towards an inverse relationship between the Danish and British MS patients with regard to haplo-
Table 2

Distribution of HRES-1 haplotypes in multiple sclerosis patients and healthy control subjects from the UK after stratification for HLADR2

\begin{tabular}{crrrrr}
\hline \multirow{2}{*}{ Haplotypes } & \multicolumn{2}{c}{ MS patients } & & \multicolumn{2}{c}{ Control subjects $^{\mathrm{a}}$} \\
\cline { 2 - 3 } \cline { 5 - 6 } & \multicolumn{1}{c}{ DR2+ } & \multicolumn{1}{c}{ DR2- } & & \multicolumn{1}{c}{ DR2+ } & \multicolumn{1}{c}{ DR2- } \\
\hline 1 & $53(0.43)$ & $41(0.42)$ & & $23(0.38)$ & $37(0.26)$ \\
2 & $30(0.25)$ & $23(0.23)$ & & $19(0.32)$ & $53(0.38)$ \\
3 & $7(0.06)$ & $6(0.06)$ & & $6(0.10)$ & $12(0.09)$ \\
4 & $32(0.26)$ & $28(0.29)$ & & $12(0.20)$ & $38(0.27)$ \\
\hline
\end{tabular}

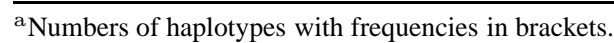

Breslow-Day test to assess homogeneity of odds ratio between MS and HRES-1 haplotype 1 in a $2 \times 2$ table stratified for DR2: chisquare value $=1.28, d f=1, p=0.26$.

type 2 and 3 . Interpreting the finding of an association between HRES-1 and MS in the UK as a false positive or spurious association would probably not be correct as relatively large numbers of patients and control subjects had been examined. Neither is the possibility likely that the finding is due to differences in the linkage disequilibrium between HRES-1 and a neighbor locus harboring a disease allele since the healthy control subjects from the two countries were comparable with respect to HRES-1 haplotype frequency. Instead, we favor the interpretation that the finding reflects existence of genetically different subsets of MS with HRES-1 being a marker of one these.

The study of a disease in different ethnic groups is often useful to distinguish the effect of a true susceptibility gene from a marker-disease associations due to linkage disequilibrium. In a previous study we have searched for an association between HRES-1 and MS in a population of Shanghai Chinese [12]. No such association was found, perhaps suggesting that different 
genes are associated with MS in populations of different ethnic derivations. Moreover, the lack of an association in the population of Shanghai Chinese hindered speculations as to how the HRES-1 locus may be affecting susceptibility to MS in populations from Northern Europe.

In conclusion, the present study have provided evidence of a weak association between a haplotype of HRES- 1 and MS in the UK. Our observations also raise the question of HRES-1 being a marker of a specific subset of MS. Large-scale studies stratifying for clinical phenotypes may help to answer this. Obviously, identification of genetic markers of different subtypes of MS would be important to the understanding of the etiology of this disease.

\section{Acknowledgments}

The Multiple Sclerosis Society in Denmark supported this study.

\section{References}

[1] K. Banki, J. Maceda, E. Hurley, E. Ablonczy, D.H. Mattson, L. Szegedy, C. Hung and A. Perl, Human T-cell lymphotropic virus (HTLV)-related endogenous sequence, HRES1, encodes a 28-kDa protein: a possible autoantigen for HTLVI gag-reactive autoantibodies, Proc Natl Acad Sci (USA) 89 (1992), 1939-1943.

[2] J. Chataway, R. Feakes, F. Coraddu, J. Gray, J. Deans, M. Fraser, N. Robertson, S. Broadley, H. Jones, D. Clayton, P. Goodfellow, S. Sawcer and A. Compston, The genetics of multiple sclerosis: principles, background and updated results of the United Kingdom systematic genome screen, Brain 121 (1998), 1869-1887.

[3] D.A.S. Compston, H. Kellar Wood, N. Robertson, S. Sawcer and N.W. Wood, Genes and susceptibility to multiple sclerosis, Acta Neurol. Scand. 161 (suppl.) (1995), 43-51.

[4] M.A. Kelly, K.H. Jacobs, M.A. Penny, C.H. Mijovic, S. Nightingale, A.H. Barnett and D.A. Francis, An investigation of HLA-encoded genetic susceptibility to multiple sclerosis in subjects of Asian Indian and Afro-Caribbean ethnic origin, Tissue Antigens 45 (1995), 197-202.

[5] M.A. Kelly, Y. Zhang, M.A. Penny, K.H. Jacobs, D.A. Cavan, C.H. Mijovic, K.-Y. Chou, A.H. Barnett and D.A. Francis, Ge- netic susceptibility to multiple sclerosis in a Shanghai Chinese population. The role of the HLA class II genes, Hum Immunol 42 (1995), 203-208.

[6] J. Kira, T. Kanai, Y. Nishimura, K. Yamasaki, S. Matsushita, Y. Kawano, K. Hasuo, S. Tobimatsu and T. Kobayashi, Western versus Asian types of multiple sclerosis: immunogenetically and clinically distinct disorders, Ann Neurol 40 (1996), 569574.

[7] C. Magistrelli, E. Samoilova, R.K. Agarwal, K. Banki, P. Ferrante, A. Vladutiu, P.E. Phillips and A. Perl, Polymorphic genotypes of the HRES-1 human endogenous retrovirus locus correlate with systemic lupus erythematosus and autoreactivity, Immunogenetics 49 (1999), 829-834.

[8] M.G. Marrosu, M.R. Murru, G. Costa, F. Cucca, S. Sotgiu, G. Rosati and F. Muntoni, Multiple sclerosis in Sardinia is associated and in linkage disequilibrium with HLA- DR3 and DR4 alleles, Am J Hum Genet 61 (1997), 454-457.

[9] C.M. Poser, D.W. Paty, L. Scheinberg, W.I. McDonald, F.A. Davis, G.C. Ebers, K.P. Johnson, W.A. Sibley, D.H. Silberberg and W.W. Tourtelotte, New diagnostic criteria for multiple sclerosis: guidelines for research protocols, Ann Neurol $\mathbf{1 3}$ (1983), 227-231.

[10] H.B. Rasmussen, A. Heltberg, K. Christensen and J. Clausen, Possible association between multiple sclerosis and the human T cell leukemia virus (HTLV)-related endogenous element, HRES-1, Mult scler 2 (1996), 133-136.

[11] H.B. Rasmussen and J. Clausen, Possible involvement of endogenous retroviruses in the development of autoimmune disorders, especially multiple sclerosis, Acta Neurol. Scand. 169 (suppl.) (1997), 32-37.

[12] H.B. Rasmussen, M.A. Kelly, D.A. Francis and J. Clausen, Haplotypes of the endogenous retrovirus HRES-1 in multiple sclerosis patients and healthy control subjects of Shanghai Chinese origin, Dis Markers 13 (1998), 251-255.

[13] H.B. Rasmussen and J. Clausen, A novel haplotype of the endogenous retrovirus, HRES-1, in patients with multiple sclerosis and healthy individuals, Autoimmunity 29 (1999), 141145.

[14] P.C. Sham and D. Curtis, Monte Carlo tests for associations between disease and alleles at highly polymorphic loci, Ann Hum Genet 59 (1995), 97-105.

[15] M. Tas, M. de Haan-Meulman, P.J. Kabel and H.A. Drexhage, Defects in monocyte polarization and dendritic cell clustering in patients with Graves' disease. A putative role for a nonspecific immunoregulatory factor related to retroviral $\mathrm{p} 15 \mathrm{E}$, Clin Endocrinol 34 (1991), 441-448.

[16] J.D. Terwilliger and J. Ott, Handbook of genetic linkage, John Hopkins University Press, Baltimore and London, 1994.

[17] M. Walchner, C. Leib-Mösch, G. Messer, H. Germaier, G. Plewig and P. Kind, Endogenous retroviral sequences in the pathogenesis of systemic autoimmune disease, Arch Dermatol 133 (1997), 767-771. 


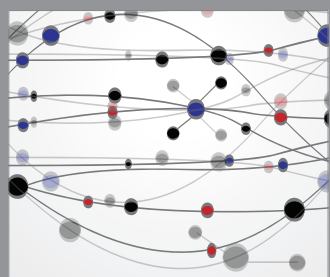

The Scientific World Journal
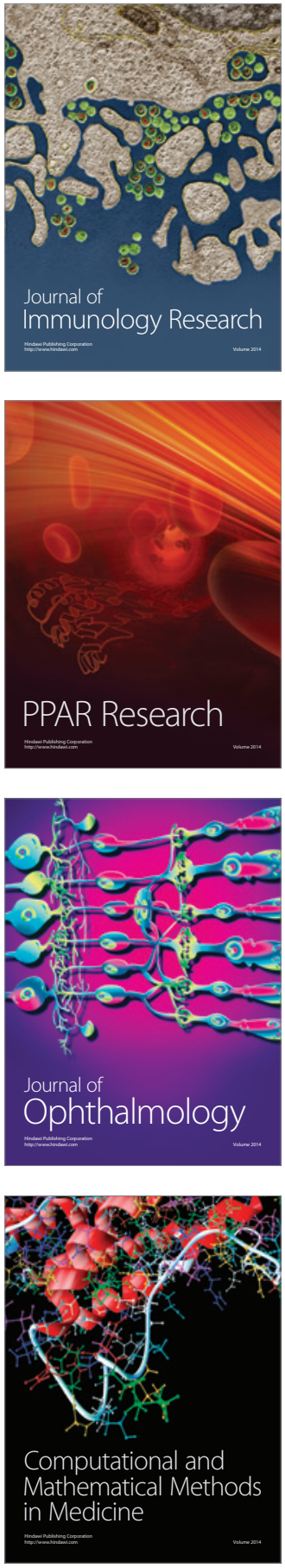

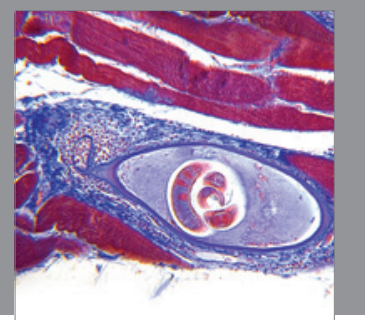

Gastroenterology

Research and Practice
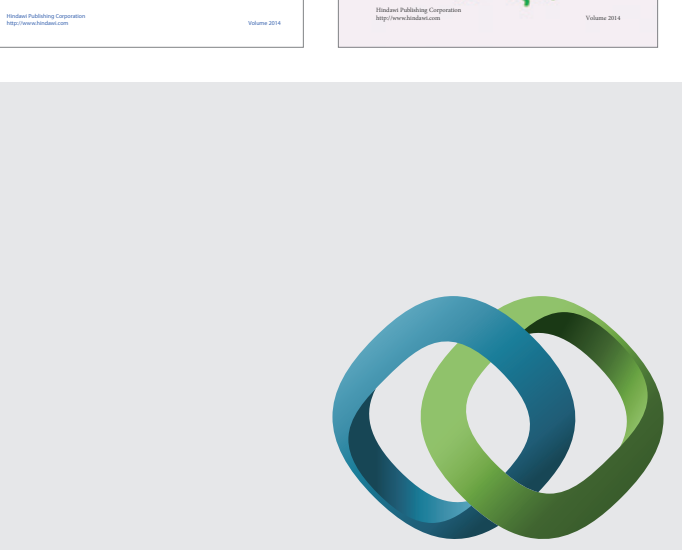

\section{Hindawi}

Submit your manuscripts at

http://www.hindawi.com
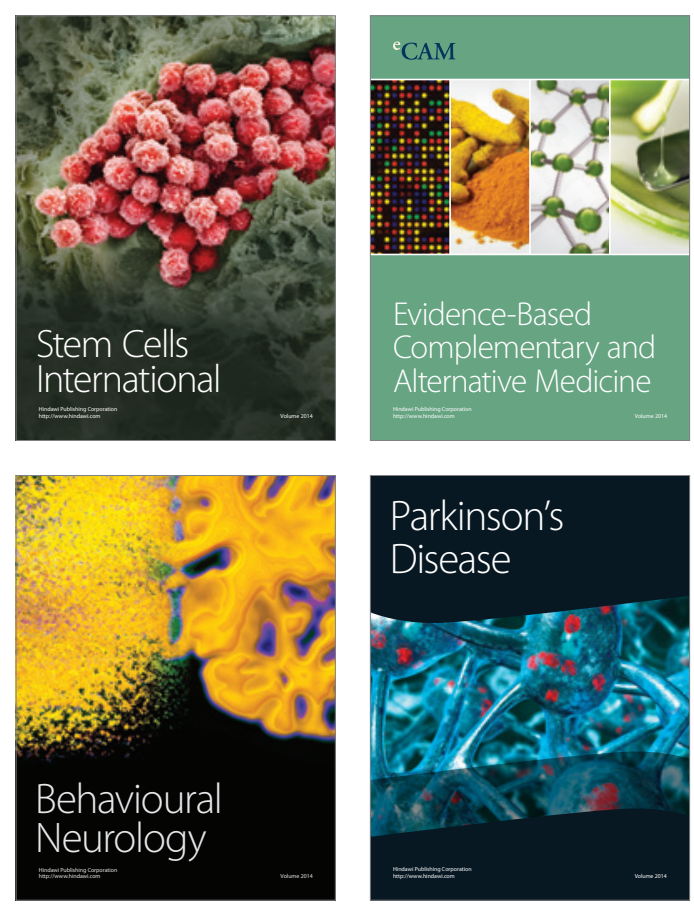

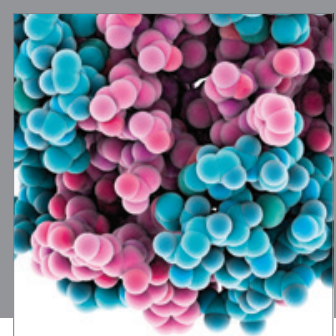

Journal of
Diabetes Research

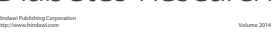

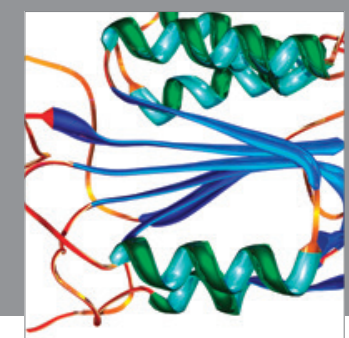

Disease Markers
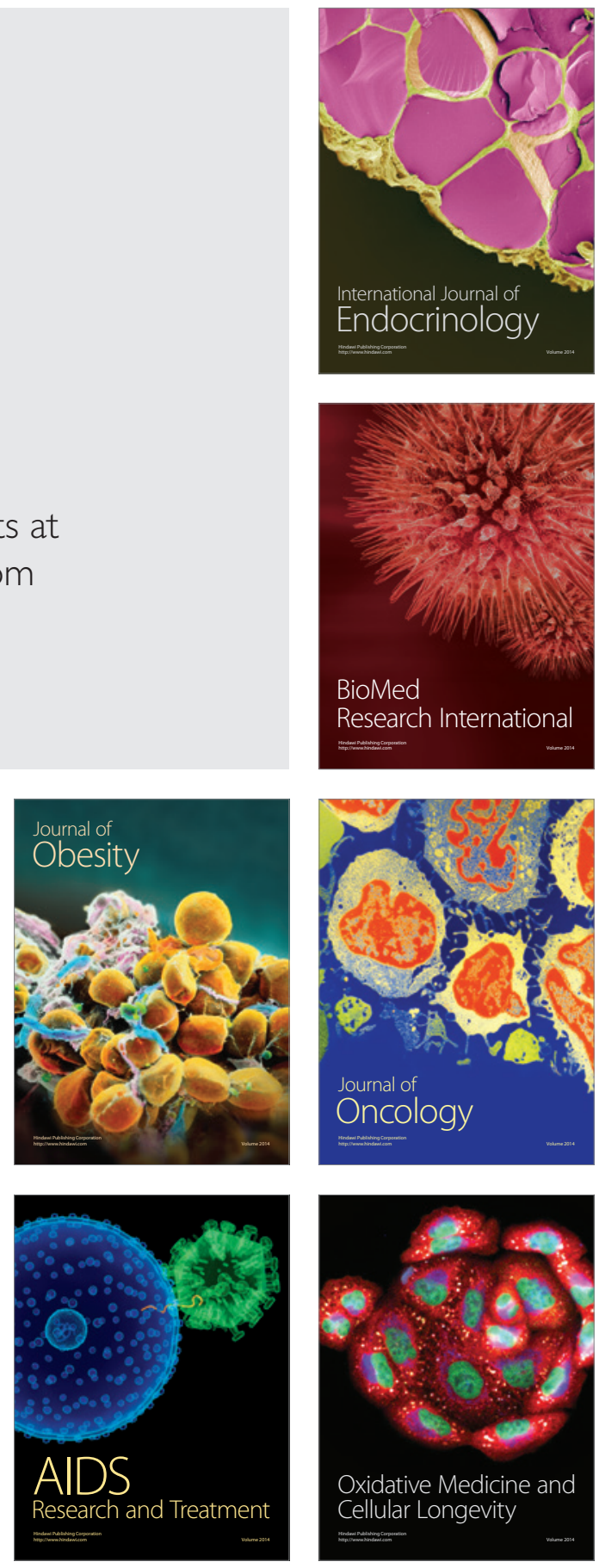